\title{
Eficiência de cultivares de milho na absorção e uso de nitrogênio em ambiente de casa de vegetação
}

\section{Efficiency of maize cultivars in the absorption and use of nitrogen in a greenhouse environment}

\author{
Rafael Pelloso de Carvalho ${ }^{1 *}$; Renzo Garcia Von Pinho²; Livia Maria Chamma Davide ${ }^{3}$
}

\section{Resumo}

Os objetivos deste trabalho foram avaliar cultivares de milho quanto à eficiência na absorção e uso de nitrogênio $(\mathrm{N})$ através de índices de eficiência de utilização de nitrogênio, em níveis contrastantes desse nutriente em ambiente de casa de vegetação. O experimento foi conduzido em casa de vegetação e o delineamento experimental utilizado foi o de blocos casualizados, com 20 tratamentos e quatro repetições, dispostos em um esquema fatorial de $10 \times 2$. As 10 cultivares de milho foram representadas por variedades locais e híbridos comerciais e as doses de $\mathrm{N}$ aplicadas foram de $100 \mathrm{e} 400 \mathrm{mg} \mathrm{dm}^{-3}$. As parcelas foram representadas por vasos de $8 \mathrm{~L}$, os quais continham 4 plantas por vaso, sendo colhidas no estádio fenológico V8. As características avaliadas foram o peso de matéria seca e teor de $\mathrm{N}$ da parte aérea e raízes. Também foi avaliada a eficiência nutricional das cultivares. As cultivares estudadas apresentaram características distintas quanto à eficiência no uso e absorção de $\mathrm{N}$ nos ambientes com alto e baixo nível de nitrogênio. Os níveis contrastantes de $\mathrm{N}$ utilizados permitiram que ocorressem diferenças necessárias para distinguir as cultivares mais eficientes. Genótipos eficientes para uso de $\mathrm{N} \mathrm{e}$ responsivos a sua aplicação podem ser selecionados pelos índices utilizados nesse trabalho.

Palavras-chave: Zea mays, casa de vegetação, doses de nitrogênio

\begin{abstract}
The objectives of this study were to evaluate corn cultivars for efficiency in the absorption and use of nitrogen (N) levels of efficiency through the use of nitrogen in contrasting levels of this nutrient in the greenhouse. The experiment was carried out in a greenhouse and the experimental design was in randomized blocks, with 20 treatments and four replicates, in a $10 \times 2$ factorial arrangement. All 10 maize cultivars were represented by local varieties and commercial hybrids, and the applied $\mathrm{N}$ levels were 100 and $400 \mathrm{mg} \mathrm{dm}^{-3}$. Plots were represented by $8 \mathrm{~L}$ pots containing 4 plants each, which were harvested in phenological stage V8. The evaluated characteristics were dry matter weight and N level of shoot and roots. The nutritional efficiency of cultivars was also evaluated. The studied cultivars had distinct characteristics concerning $\mathrm{N}$ use and absorption efficiency in environments of high and low nitrogen levels. The contrasting $\mathrm{N}$ levels allowed the occurrence of differences needed to distinguish the most efficient cultivars. Genotypes efficient for $\mathrm{N}$ use and responsive to its application can be selected based on the indexes used in this work.
\end{abstract}

Key words: Zea mays, greenhouse, nitrogen levels

\footnotetext{
${ }^{1}$ Eng $^{\circ}$ Agr $^{\circ}$, MSc. em Fitotecnia, Doutorando do Programa de Pós Graduação em Produção Vegetal da Universidade Federal da Grande Dourados, UFGD. Faculdade de Ciências Agrárias, FCA, Dourados, MS. E-mail: pellosodecarvalho@yahoo.com.br

${ }^{2}$ Eng $^{\circ}$ Agr $^{\circ}$, Prof. Dr. Adjunto do Dept ${ }^{\circ}$ de Fitotecnia da Universidade Federal de Lavras, UFLA, Lavras, MG. E-mail: renzo@dag. ufla.br

3 Eng ${ }^{\circ}$ Agr $^{\circ}$, Prof. Dr. Adjunto da UFGD/FCA, Dourados, MS. E-mail: liviadavide@ufgd.edu.br

* Autor para correspondência
} 


\section{Introdução}

Visando atender a crescente demanda mundial de alimentos e desenvolver soluções tecnológicas que levem a redução do risco associado à atividade agrícola tem-se buscado uma maior eficiência no uso do nitrogênio em solos tropicais. A obtenção de maior eficiência no uso desse elemento tem sido um objetivo almejado tanto para a agricultura capitalizada, quanto para a de baixos insumos. Isto porque, os desperdícios e a escassez do nitrogênio podem gerar problemas econômicos, ambientais, de saúde pública e de segurança alimentar.

Várias estratégias podem ser tomadas com intuito de aumentar a eficiência no uso de $\mathrm{N}$, tais como a redução das doses de adubos para níveis que sejam produtivos e seguros; a utilização de leguminosas em rotação, que fornecem $\mathrm{N}$ e melhoram várias características do solo; a fixação biológica de $\mathrm{N}$ em gramíneas e a agricultura de precisão. Outra estratégia é a seleção e/ou o desenvolvimento de cultivares com maior eficiência no uso de nitrogênio.

Acredita-se que a eficiência no uso de nitrogênio seja um caráter complexo dependente de vários processos fisiológicos tais como absorção, assimilação e retranslocação do nitrogênio pela planta, e o seu melhor entendimento poderá colaborar com possíveis soluções para problemas associadas à falta e ao excesso de nitrogênio na agricultura.

Em geral, a eficiência nutricional pode expressar a relação entre a produção obtida e os insumos aplicados. Isto significa que a eficiência nutricional é a quantidade de matéria seca ou grãos produzidos por unidade de nutriente aplicado. Entretanto, na literatura, a eficiência nutricional é definida de várias maneiras, dividindo-se basicamente entre aquelas que consideram o requerimento interno do nutriente na planta, e aquelas que enfatizam a produtividade (GOURLEY; ALLAN; RUSSELE, 1994).

Sob o ponto de vista agronômico, a eficiência nutricional refere-se à eficiência de uma espécie ou genótipo em gerar altas produtividades num solo deficiente em determinado nutriente (GRAHAM, 1984). Sob o ponto de vista fisiológico seria a capacidade de um genótipo absorver o nutriente do solo, distribuí-lo e utilizá-lo em diferentes processos fisiológicos (GODDARD; HOLLIS, 1984).

Dependendo do nutriente, deve ser também considerada a eficiência de sua redistribuição interna (MOLL; KAMPRATH; JACKSON, 1982). Segundo Clark (1982), uma planta eficiente pode ser aquela que produza o maior peso seco total ou o maior peso seco da fração a ser colhida por unidade de nutriente absorvido pela planta; havendo dois aspectos principais a considerar: a eficiência com que as plantas recuperam os fertilizantes aplicados e a eficiência com a qual as plantas usam os elementos após a sua absorção.

Cooke (1987) define ainda a eficiência nutricional como sendo o aumento da produtividade por unidade de nutriente aplicado. Já para Israel e Rufty Júnior (1988) a eficiência nutricional é a relação entre a biomassa total e a quantidade de nutriente absorvido.

Às vezes, na literatura, a eficiência nutricional é também chamada de eficiência de absorção. Outras denominações podem ser encontradas, como por exemplo, eficiência de enraizamento e de conversão de biomassa (MARTINEZ et al., 1993). A eficiência de absorção é a quantidade de nutriente absorvido por unidade de matéria seca das raízes. A eficiência de enraizamento é a produção de matéria seca das raízes por unidade de nutriente absorvida; já a eficiência de conversão em biomassa é a produção de matéria seca da parte aérea por unidade de nutriente na parte aérea (MARANVILLE; CLARK; ROSS, 1980).

A melhor eficiência nutricional é aquela obtida sob nível de nutriente adequado em que à produtividade máxima foi obtida e a relação de eficiência deve estar relacionada à parte da planta de interesse econômico para que não se selecione plantas com alta eficiência de absorção e baixa produção econômica. Portanto, deve-se tomar 
cuidado na interpretação de resultados de eficiência nutricional (FAGERIA, 1998).

Especificamente em milho, Moll, Kamprath e Jackson (1982) definiram a eficiência no uso do N (EUN) como a massa de grãos dividida pela massa de $\mathrm{N}$ aplicado no solo (Gw/Ns), ambas expressas na mesma unidade, como por exemplo, gramas por planta. Definiram também, dois componentes primários desta eficiência, que são chamados de eficiência na absorção e eficiência na utilização. O primeiro é a quantidade de $\mathrm{N}$ total na planta na maturidade dividido pelo $\mathrm{N}$ aplicado no solo (NT/ $\mathrm{Ns}$ ), e o segundo é a massa de grãos dividida pelo $\mathrm{N}$ total da planta na maturidade $(\mathrm{Gw} / \mathrm{Nt})$. A EUN seria o produto da eficiência na absorção pela eficiência na utilização. Neste trabalho os autores compararam vários materiais genéticos de milho e mostram que os componentes da EUN podem ser diferentes mesmo entre híbridos com similar EUN total.

Diferenças genotípicas quanto à absorção, translocação e acumulação de $\mathrm{NO}_{3}^{-}$em híbridos e linhagens de milho foram observadas por diversos autores (YOUNGQUIST; COX; MARANVILLE, 1992; MARRIEL et al., 2000; FERNANDES; BUZZETI;ANDRADE, 2005).

Dessa forma o presente estudo, objetivou-se avaliar cultivares de milho quanto à eficiência na absorção e uso de nitrogênio através de índices de eficiência de utilização de nitrogênio, em níveis contrastantes desse nutriente em ambiente de casa de vegetação.

\section{Material e Métodos}

O experimento foi conduzido em casa de vegetação localizada na área experimental do Departamento de Ciência do Solo da Universidade Federal de Lavras (UFLA).

Foram utilizadas 10 cultivares comerciais de milho, entre variedades locais e híbridos comerciais, recomendados comercialmente para três níveis de investimento e que se mostram contrastantes quanto à produção de grãos, em condições normais de cultivo (Tabela 1).

Tabela 1. Características das cultivares de milho utilizadas para avaliação da eficiência de absorção e uso de nitrogênio em casa de vegetação.

\begin{tabular}{llcl}
\hline Material Genético & Empresa & Classificação & Investimento \\
\hline P30F53 & Pioneer & Híbrido Simples & Alto* \\
GNZ2004 & Geneze & Híbrido Simples & Alto \\
DKB390 & Dekalb & Híbrido Simples & Alto \\
AG5020 & Agroceres & Híbrido Triplo & Médio** \\
DKB566 & Dekalb & Híbrido Triplo & Médio \\
P30S40 & Pioneer & Híbrido Triplo & Médio \\
DKB798 & Dekalb & Híbrido Duplo & Baixo*** \\
BM2202 & Biomatrix & Híbrido Duplo & Baixo \\
DKB747 & Dekalb & Híbrido Duplo & Baixo \\
AL25 & CATI & Variedade & Baixo \\
\hline
\end{tabular}

*Alto potencial produtivo, alta exigência tecnológica e preço da semente acima de $\mathrm{R} \$ 180,00$; **Médio potencial produtivo, média exigência tecnológica e preço da semente de $\mathrm{R} \$ 100,00$ até $\mathrm{R} \$ 180,00$; ***Baixo potencial produtivo, baixa exigência tecnológica e preço da semente até $\mathrm{R} \$ 100,00$.

Fonte: Elaboração dos autores. 
Os tratamentos, dispostos em um esquema fatorial $10 \times 2$, sendo 10 genótipos de milho e duas doses de $\mathrm{N}$ (100 e $400 \mathrm{mg} \mathrm{dm}^{-3}$ de $\mathrm{N}$ ), foram distribuídos no delineamento blocos casualizados, com quatro repetições, totalizando 80 parcelas experimentais.

As parcelas foram representadas por vasos com capacidade de $8 \mathrm{~L}$, preenchidos com uma mistura de terra seca ao ar, peneiradas em malha de $5 \mathrm{~mm}$ e areia lavada, na proporção de $3: 1$, respectivamente. A coleta da terra foi realizada em subsolo classificado como Latossolo Vermelho Distroférrico Típico LVdf (EMBRAPA, 1999), localizado no campus universitário da UFLA e submetida a análise química e granulométrica do solo.

O resultado da análise granulométrica permitiu classificar o solo como muito argiloso, fornecendo um resultado em dag $\mathrm{Kg}^{-1}$ de 18, 16 e 66 para areia, silte e argila, respectivamente. Através deste resultado foi tomada a decisão da mistura solo e areia, visando melhorar a infiltração de água durante a irrigação dos vasos. A análise química do solo, realizada antes da adição da areia, revelou as seguintes características: $\mathrm{pH}$ (água) $=5,9 ; \mathrm{P}$ e K $\left(\mathrm{mg} \cdot \mathrm{dm}^{-3}\right)=0,4$ e 8,$0 ; \mathrm{Ca}, \mathrm{Mg}$ e $\mathrm{H}+\mathrm{Al}\left(\mathrm{cmol}_{\mathrm{c}} \cdot \mathrm{dm}^{-3}\right)$ $=0,2 ; 0,1$ e 1,3; $\mathrm{MO}\left(\right.$ dag. $\left._{\mathrm{kg}}{ }^{-1}\right)=0,2 ; \mathrm{SB}\left(\mathrm{cmol}_{\mathrm{c}} \mathrm{dm}^{-}\right.$ $\left.{ }^{3}\right)=0,3$ e $\mathrm{V}(\%)=19,8$.

Com base nos resultados da análise química do solo realizou-se a calagem, transformando-se as doses obtidas em $\mathrm{Kg}$ ha $^{-1}$ recomendada a campo para $\mathrm{Kg}$ de solo puro adicionado por vaso. Para efeito dos cálculos da calagem não foi considerado o volume de areia misturada ao solo utilizado. $\mathrm{O}$ método utilizado foi o de saturação por bases (SB), elevando a saturação ao nível de 70\%. O calcáreo utilizado foi o dolomítico com PRNT de 90\%. A quantidade requerida de calcário foi de 0,44 gramas por Kg de solo aplicado. Após a aplicação e homogeneização deste calcáreo ao solo seco, fez-se a mistura com areia e posterior preenchimento dos vasos, os quais foram submetidos a uma incubação por um período de aproximadamente 20 dias, sendo regularmente irrigado com o intuito de mantê-lo com $70 \%$ da máxima capacidade de retenção de água, suficiente para a reação.

O crescimento do milho foi avaliado nas condições de baixa e alta disponibilidade de $\mathrm{N}$ e sem limitação dos demais nutrientes. Nas parcelas submetidas a alto nível de $\mathrm{N}$ foi aplicado $400 \mathrm{mg}$ $\mathrm{dm}^{-3}$ do nutriente. Já nas parcelas com baixo nível de $\mathrm{N}$ foi aplicado $100 \mathrm{mg} \mathrm{dm}^{-3}$ do nutriente.

A adubação nitrogenada foi parcelada em três vezes, sendo a primeira no plantio, onde foram aplicados $100 \mathrm{mg} \mathrm{dm}^{-3}$ de $\mathrm{N}$ para todos os tratamentos. Nas parcelas que correspondiam ao alto nível de $\mathrm{N}$, o restante foi dividido em duas coberturas de $150 \mathrm{mg} \mathrm{dm}^{-3}$ e aplicados nos estádios $\mathrm{V} 3$ e V6. A fonte de $\mathrm{N}$ utilizada foi a uréia, contendo $45 \%$ de $\mathrm{N}$.

As quantidades dos outros nutrientes essenciais a cultura do milho foram fornecidos e ajustados de acordo com as recomendações de Malavolta (1980), com pequenas modificações. Os nutrientes foram aplicados na dose recomendada, na forma de solução nutritiva por meio de pipeta, ao solo seco, fazendose em seguida, uma cuidadosa homogeneização.

Após a calagem e adubação do solo para plantio, foi realizada nova amostragem de solo para análise química, que revelou as seguintes características: $\mathrm{pH}($ água $)=6,1 ; \mathrm{P}$ e K $\left(\mathrm{mg} \cdot \mathrm{dm}^{-3}\right)=5,7$ e 70,0; Ca, $\mathrm{Mg} \mathrm{e} \mathrm{H}+\mathrm{Al}\left(\mathrm{cmol}_{\mathrm{c}} \cdot \mathrm{dm}^{-3}\right)=2,3 ; 0,6$ e 1,$3 ; \mathrm{MO}(\mathrm{dag}$ . $\left.\mathrm{kg}^{-1}\right)=0,3 ; \mathrm{SB}\left(\mathrm{cmol}_{\mathrm{c}} \mathrm{dm}^{-3}\right)=3,1$ e $\mathrm{V}(\%)=70,3$.

$\mathrm{O}$ experimento foi conduzido durante período de inverno (junho/julho) com o intuito de propiciar temperaturas mais amenas no interior da casa de vegetação, visto que no verão as temperaturas sobem consideravelmente proporcionando um estiolamento acentuado das plantas de milho. As temperaturas máxima e mínima observadas no interior da casa de vegetação durante o período de desenvolvimento das plantas de milho foram respectivamente, $28^{\circ} \mathrm{C}$ e $9^{\circ} \mathrm{C}$. Podendo haver uma amplitude de variação de $2^{\circ} \mathrm{C}$ para mais ou para menos para ambas as temperaturas. 
Foram semeadas oito sementes por vaso e, após a emergência das plântulas, foi efetuado o desbaste, deixando-se quatro plantas por vaso.

Para fins de irrigação, foi mantida a umidade entre $50 \%$ e $70 \%$ da capacidade máxima de retenção de água pelo solo por meio de irrigações controladas por pesagem dos vasos.

Todas as plantas foram colhidas no dia 15 de julho, no estádio V8, lavadas em água destilada e separadas em raízes e parte aérea para determinação da massa verde total de cada uma das partes. Este material foi colocado em estufa com circulação forçada de ar a $75^{\circ} \mathrm{C}$ até atingir peso constante e pesados em balança analítica para determinação de peso seco de raízes e parte aérea. Na seqüência, as amostras foram moídas e submetidas às análises laboratoriais para determinação do teor de nitrogênio de raízes e parte aérea, usando o método estabelecido por Kjeldahl e descrito em Melo (1977). Os valores de acúmulo de $\mathrm{N}$ foram obtidos por meio do produto entre o teor de $\mathrm{N}$ na planta e o peso de matéria seca (MS).

Os dados obtidos foram submetidos à análise de variância, e as médias agrupadas pelo teste de Scott e Knott (1974), a 1\% e 5\% de probabilidade, utilizando o aplicativo computacional SISVAR (FERREIRA, 1999).

Para avaliar a eficiência nutricional das cultivares foram utilizados os métodos proposto por Fageria e Kluthcouski (1980) e Chun et al. (2005), os quais se baseiam na representação da posição gráfica das cultivares no plano cartesiano a fim de classificálas quanto à eficiência nutricional e a resposta ao nitrogênio.

\section{Resultados e Discussão}

Peso de matéria seca de parte aérea e do sistema radicular

De acordo com o teste de $\mathrm{F}$ as diferentes doses de N (100 e $400 \mathrm{mg} \mathrm{dm}^{-3}$ ) utilizadas apresentaram efeitos semelhantes para o peso de matéria seca de parte aérea e de raiz (Tabela 2). Esse resultado pode ser explicado por meio da dose utilizada para o ambiente em baixo N. A menor dose de nitrogênio empregada não foi suficiente para que ocorresse a deficiência de $\mathrm{N}$ a ponto de alterar a morfologia das plantas. Presterl et al. (2003) demonstraram que em ambientes com estresse de $\mathrm{N}$ ocorre o atraso do alongamento do caule e do crescimento de folhas, aumentando ainda o crescimento de raízes. Portanto, a menor dose utilizada de $\mathrm{N}$ proporcionou um desenvolvimento relativo das raízes e da parte aérea, insuficiente para que fosse detectada diferença significativa entre a maior e menor dose de N.

Para o peso de matéria seca de parte aérea (MSPA) foi constatada diferença significativa entre as cultivares (Tabela 2). As cultivares DKB747, DKB789 e AL25, não diferiram entre si, e foram inferiores aos demais genótipos avaliados (Tabela 3). As outras cultivares foram semelhantes entre si, acumulando, em média, patamares de até 33,47 g de MSPA, alcançando uma diferença de $37 \%$ em relação a menor média obtida.

Essa constatação demonstra a variabilidade genética entre os genótipos testados para esta característica, indicando comportamento diferenciado entre as cultivares tanto em ambientes com baixa, como com alta disponibilidade de $\mathrm{N}$.

Com relação ao peso de matéria seca do sistema radicular (MSR), verificou-se diferença significativa $(\mathrm{p} \leq 0,05)$ para a interação cultivares $\mathrm{x}$ doses, permitindo concluir que as doses de 100 e $400 \mathrm{mg}$ $\mathrm{dm}^{-3}$ de $\mathrm{N}$ resultaram em diferentes efeitos sobre as dez cultivares estudadas (Tabela 2). Analisando a interação e desdobrando as doses dentro de cada nível de cultivar, pode-se verificar que não houve diferença significativa para o peso de MSR quando utilizou-se as doses para qualquer cultivar. Entretanto, quando realizou o desdobramento de cultivar dentro de cada nível de N, observou-se que as dez cultivares têm efeitos diferentes sobre a dose $100 \mathrm{mg} \mathrm{dm}^{-3} \mathrm{e}$ também sobre a dose de $400 \mathrm{mg} \mathrm{dm}^{-3}$. 
Tabela 2. Resumo da análise de variância para o acúmulo de matéria seca na parte aérea (MSPA), no sistema radicular (MSR), para a relação parte áerea/raiz (PA/R), para os teores de $\mathrm{N}$ na parte aérea (\%NPA) e para os teores de $\mathrm{N}$ na raiz $(\% \mathrm{NR})$.

\begin{tabular}{lccccc}
\hline Fontes de & \multicolumn{5}{c}{ Quadrados Médios } \\
\cline { 2 - 6 } Variação & MSPA & MSR & PA/R & \%NPA & \%NR \\
\hline Bloco & 122,19 & 0,16 & 2,54 & 63,58 & 92,50 \\
Cultivar (C) & $138,98^{* *}$ & $49,16^{* *}$ & $9,18^{* *}$ & $45,86^{* *}$ & 8,49 \\
Doses (D) & 187,43 & 0,33 & $7,48^{* *}$ & $1193,51^{* *}$ & $72,20^{* *}$ \\
C x D & 105,60 & $13,69^{* *}$ & 5,12 & 6,37 & 5,39 \\
C d. D (100) & - & $19,91^{*}$ & - & - & - \\
C d. D (400) & - & $42,94^{*}$ & - & - & - \\
Erro & 62,97 & 6,34 & 1,56 & 11,93 & 11,59 \\
\hline CV (\%) & 28,53 & 24,04 & 41,17 & 9,26 & 22,85 \\
Média & 27,81 & 10,47 & & 37,29 & 14,90 \\
\hline
\end{tabular}

** significativo a $5 \%$ de probabilidade pelo teste $\mathrm{F}$.

Fonte: Elaboração dos autores.

No ambiente com baixo $\mathrm{N}$, as cultivares DKB390, BM2202, AG5020, DKB747, DKB566, P30S40 e DKB789 foram superiores às cultivares AL25, P30F53 e GNZ2004, as quais não diferiram entre si (Tabela 3). Neste ambiente, os valores de MSR variaram de 6,39 a 13,11 gramas, atingindo diferenças de até $51 \%$. Já no ambiente com alto N, as cultivares 30S40, P30F53, DKB789, DKB747 e DKB566, não diferiram entresia 5\% de probabilidade e foram superiores às demais, apresentando valores de 5,11 até $15,25 \mathrm{~g}$, diferenciando-se em $66 \%$. Esta maior diferença apresentada pelo ambiente de alto $\mathrm{N}$, pode ser explicada pela menor tolerância que alguns materiais apresentam quanto ao teor de $\mathrm{NH}_{4}^{+}$ disponível para as plantas. Provavelmente, a maior quantidade de $\mathrm{N}$ na forma amoniacal na solução do solo, estabelecida pelo fornecimento de altas doses de $\mathrm{N}$ pela uréia, reduziu o crescimento de raízes daquelas cultivares que apresentaram os menores valores.

Outro ponto importante a ser considerado com respeito a estas diferenças observadas é em relação entre o acesso ao nutriente limitante $(\mathrm{N})$ e a morfologia do sistema radicular. Assim, se existir estresse de $\mathrm{N}$, fatores relacionados à morfologia de raiz passam a ser mais importantes, conferindo a planta de milho um maior desenvolvimento radicular. Ao passo que, se o acesso à superfície radicular não for limitante, o mecanismo de absorção passa ser o fator principal no processo de aquisição do nutriente. Neste caso não se verifica grandes aumentos na produção de raízes (GERLOFF, 1977).

Guimarães et al. (2006), observaram diferenças de respostas entre os genótipos quando cultivados em níveis contrastantes de $\mathrm{N}$, sendo verificado pequeno desenvolvimento de parte aérea e grande desenvolvimento de raízes no experimento com baixa disponibilidade de $\mathrm{N}$, enquanto que, no ensaio com alto nitrogênio foi verificado comportamento inverso, com grande desenvolvimento de parte aérea e pequeno crescimento de raízes. 
Tabela 3. Valores médios do peso de matéria seca da parte aérea (MSPA), do sistema radicular (MSR) e da relação entre parte aérea e raiz (PA/R), nas doses de 100 e $400 \mathrm{mg} \mathrm{dm}^{-3}$ de nitrogênio.

\begin{tabular}{lccccccc}
\hline & \multicolumn{3}{c}{ MSPA (g) } & \multicolumn{2}{c}{ MSR (g) } & \multicolumn{2}{c}{ PA/R } \\
\cline { 2 - 7 } \multicolumn{1}{c}{ Cultivares } & \multicolumn{2}{c}{ Doses } & \multicolumn{1}{c}{ Doses } & \multicolumn{2}{c}{ Doses } \\
\cline { 2 - 7 } & $\mathbf{1 0 0}$ & $\mathbf{4 0 0}$ & Média & $\mathbf{1 0 0}$ & $\mathbf{4 0 0}$ & $\mathbf{1 0 0}$ & $\mathbf{4 0 0}$ \\
\hline P30F53 & 22,09 & 32,3 & $27,2 \mathrm{~B}$ & $8,37 \mathrm{~A}$ & $11,41 \mathrm{~B}$ & $2,79 \mathrm{~A}$ & $2,95 \mathrm{~B}$ \\
GNZ2004 & 21,11 & 35,12 & $28,12 \mathrm{~B}$ & $8,63 \mathrm{~A}$ & $5,11 \mathrm{~A}$ & $2,59 \mathrm{~A}$ & $7,38 \mathrm{C}$ \\
DKB390 & 33,92 & 31,4 & $32,66 \mathrm{~B}$ & $9,98 \mathrm{~B}$ & $7,24 \mathrm{~A}$ & $3,66 \mathrm{~A}$ & $4,43 \mathrm{~B}$ \\
AG5020 & 34,45 & 32,49 & $33,47 \mathrm{~B}$ & $10,98 \mathrm{~B}$ & $9,3 \mathrm{~A}$ & $3,20 \mathrm{~A}$ & $3,54 \mathrm{~B}$ \\
DKB566 & 27,14 & 32,7 & $29,92 \mathrm{~B}$ & $12,38 \mathrm{~B}$ & $15,25 \mathrm{~B}$ & $2,28 \mathrm{~A}$ & $2,24 \mathrm{~A}$ \\
P30S40 & 22,1 & 33,47 & $27,79 \mathrm{~B}$ & $12,97 \mathrm{~B}$ & $10,93 \mathrm{~B}$ & $1,68 \mathrm{~A}$ & $3,19 \mathrm{~B}$ \\
DKB789 & 22,27 & 22,81 & $22,54 \mathrm{~A}$ & $13,11 \mathrm{~B}$ & $13,77 \mathrm{~B}$ & $1,72 \mathrm{~A}$ & $1,63 \mathrm{~A}$ \\
BM2202 & 35,67 & 26,14 & $30,91 \mathrm{~B}$ & $10,4 \mathrm{~B}$ & $7,94 \mathrm{~A}$ & $3,76 \mathrm{~A}$ & $3,54 \mathrm{~B}$ \\
DKB747 & 20,66 & 20,97 & $20,82 \mathrm{~A}$ & $12,18 \mathrm{~B}$ & $14,07 \mathrm{~B}$ & $1,73 \mathrm{~A}$ & $1,49 \mathrm{~A}$ \\
AL25 & 23,4 & 26,04 & $24,72 \mathrm{~A}$ & $6,39 \mathrm{~A}$ & $9,09 \mathrm{~A}$ & $3,83 \mathrm{~A}$ & $2,96 \mathrm{~B}$ \\
\hline Média & $26,28 \mathrm{a}$ & $29,34 \mathrm{a}$ & & $10,54 \mathrm{a}$ & $10,41 \mathrm{a}$ & $2,72 \mathrm{a}$ & $3,34 \mathrm{~b}$ \\
\hline
\end{tabular}

Médias seguidas da mesma letra maiúscula na coluna e minúscula na linha, dentro de cada variável não diferem entre si, ao nível de 5\% pelo teste de Scott Knott.

Fonte: Elaboração dos autores.

Na literatura são observados vários trabalhos que concordam os resultados obtidos por Guimarães et al. (2006), em que verifica-se uma tendência entre as cultivares de aumentar a quantidade de MSR em ambiente com estresse de nitrogênio (FISHER; DUNHAM, 1984; PRESTERL et al., 2003). Esses resultados podem ser explicados pelo fato de as plantas de milho, ao serem submetidas ao estresse de $\mathrm{N}$, utilizarem este nutriente próximo aos sítios de absorção, ou seja, nas células das próprias raízes, a fim de desenvolverem um maior volume radicular visando aumentar a área a ser explorada. Segundo Föhse, Claassen e Jungk (1988), as raízes se tornam drenos preferenciais de fotoassimilados quando alguns nutrientes limitam o crescimento das plantas, principalmente nitrogênio e potássio, o que pode ser uma estratégia de adaptação desenvolvida para aumentar a eficiência de absorção quando há limitação de N.

No presente trabalho, os híbridos DKB789 e DKB747 apresentaram comportamento semelhante ao descrito por Guimarães et al. (2006), para ambiente de baixo N e os híbridos GNZ2004, DKB390, AG5020 e BM2202, para ambiente alto N. Os híbridos DKB566 e P30S40 se destacaram por apresentarem alto peso de MS de raiz e de parte aérea nos dois ambientes estudados. A cultivar AL25 mostrou comportamento inverso, obtendo baixo peso de MS de raiz e parte aérea nos dois ambientes (Tabela 3). Sendo assim, verifica-se que a peso de MSR e de MSPA obtidos em ambientes de alto e baixo $\mathrm{N}$ variou com a cultivar e que o fornecimento de $\mathrm{N}$ favoreceu o acúmulo de MS de raiz e parte aérea.

A diferença observada entre os resultados citados na literatura e os obtidos nesse trabalho podem ser atribuídas a dosagem de $\mathrm{N}$ utilizadas em ambientes com baixo estresse. Nesse trabalho foi usada a dose de $100 \mathrm{mg} \mathrm{dm}^{-3}$ de $\mathrm{N}$, enquanto que nos trabalhos citados não ocorreu o fornecimento de $\mathrm{N}$.

\section{Teor de nitrogênio na parte aérea e raiz}

Não foi verificada diferença significativa para a interação cultivares $\mathrm{x}$ doses para o teor de $\mathrm{N}$ na parte aérea e no sistema radicular (Tabela 2). Pode-se inferir que os efeitos das cultivares sobre os teores de nitrogênio da parte aérea (\%NPA) e da raiz (\%NR) do milho foram independentes das doses de $\mathrm{N}$ aplicadas. Também não foi verificada 
diferença significativa entre as cultivares para o $\% \mathrm{NR}$. Entretanto, as cultivares diferiram entre si, a $5 \%$ de probabilidade, para o $\%$ NPA. As cultivares AL25 e GNZ2004 apresentaram valores médios próximos a $33 \mathrm{~g} \mathrm{Kg}^{-1}$ de $\mathrm{N}$ e foram inferiores as demais cultivares, que acumularam entre 37 e $40 \mathrm{~g}$ $\mathrm{Kg}^{-1}$ (Tabela 4). Entre essas, destacou-se o híbrido
DKB789, o qual apresentou o maior \%NPA. O mesmo híbrido apresentou o maior peso de MSR em dose baixa de $\mathrm{N}$, destacando-se também na dose alta para esta característica. Portanto, esta cultivar possui diversas características positivas do ponto de vista morfológico, indicando ser promissora para maior eficiência nutricional.

Tabela 4. Valores médios do teor de teor de nitrogênio da parte aérea e do sistema radicular, nas doses de 100 e 400 $\mathrm{mg} \mathrm{dm}{ }^{-3}$ de nitrogênio.

\begin{tabular}{lcccccc}
\hline \multirow{2}{*}{ Cultivares } & \multicolumn{2}{c}{ Teor de N Parte Aérea $\mathbf{( g ~ k g )}$} & \multicolumn{3}{c}{ Teor de N Raizes (g/kg) } \\
\cline { 2 - 7 } & $\mathbf{1 0 0}$ & $\mathbf{4 0 0}$ & Média & $\mathbf{1 0 0}$ & $\mathbf{4 0 0}$ & Média \\
\hline P30F53 & 36,25 & 42,50 & $39,38 \mathrm{~B}$ & 12,00 & 14,25 & $13,13 \mathrm{~A}$ \\
GNZ2004 & 29,25 & 37,75 & $33,50 \mathrm{~A}$ & 12,50 & 17,25 & $14,88 \mathrm{~A}$ \\
BKB 390 & 32,75 & 41,50 & $37,13 \mathrm{~B}$ & 15,25 & 14,75 & $15,00 \mathrm{~A}$ \\
AG5020 & 31,75 & 42,75 & $37,25 \mathrm{~B}$ & 14,75 & 15,00 & $14,88 \mathrm{~A}$ \\
DKB566 & 36,25 & 42,75 & $39,50 \mathrm{~B}$ & 14,50 & 18,75 & $16,63 \mathrm{~A}$ \\
P30S40 & 33,25 & 43,25 & $38,25 \mathrm{~B}$ & 14,00 & 15,25 & $14,63 \mathrm{~A}$ \\
DKB789 & 37,00 & 43,00 & $40,00 \mathrm{~B}$ & 13,50 & 14,50 & $14,00 \mathrm{~A}$ \\
BM2202 & 34,75 & 40,75 & $37,75 \mathrm{~B}$ & 13,50 & 14,75 & $14,13 \mathrm{~A}$ \\
DKB747 & 34,00 & 40,50 & $37,25 \mathrm{~B}$ & 14,75 & 16,75 & $15,75 \mathrm{~A}$ \\
AL25 & 29,00 & 36,75 & $32,88 \mathrm{~A}$ & 14,75 & 17,25 & $16,00 \mathrm{~A}$ \\
\hline Média & $33,43 \mathrm{a}$ & $41,15 \mathrm{~b}$ & & $13,95 \mathrm{a}$ & $15,85 \mathrm{~b}$ & \\
\hline
\end{tabular}

Médias seguidas da mesma letra maiúscula na coluna e minúscula na linha, dentro de cada variável não diferem entre si, ao nível de 5\% pelo teste de Scott Knott.

Fonte: Elaboração dos autores.

A dose de $400 \mathrm{mg} \mathrm{dm}^{-3}$ foi superior à de $100 \mathrm{mg}$ $\mathrm{dm}^{-3}$ sobre os teores de $\mathrm{N}$ da parte aérea do milho (Tabela 4). O mesmo resultado foi encontrado para os teores de $\mathrm{N}$ na matéria seca de raiz. Portanto, as variações nos teores de nitrogênio na parte aérea e raiz foram diretamente proporcionais aos níveis de nitrogênio.

Os teores de $\mathrm{N}$ na MS da parte aérea das cultivares foram sempre maiores do que os teores de na MS de raízes. Esses valores eram esperados, pois a relação de produção de MS entre parte aérea/ raiz foi sempre positiva, obtendo neste experimento uma relação média para alto nível de $\mathrm{N}$ de 3,33 e para baixo $\mathrm{N}$ de 2,72 (Tabela 4).

Analisando o teor de $\mathrm{N}$ nas raízes das cultivares e considerando que não houve diferença entre elas, pode-se sugerir que algumas cultivares mostraram- se mais eficientes em utilizar o nitrogênio absorvido, convertendo-o em tecido vegetal através de processos metabólicos ocorridos em nível celular, aumentando seu volume radicular. Enquanto que outras cultivares absorveram relativamente à mesma quantidade de $\mathrm{N}$ das demais só que não reverteram este nutriente em energia para crescimento radicular e sim acumularam o nitrogênio principalmente nos vacúolos contidos no interior das células vegetais.

\section{Avaliação da eficiência nutricional das cultivares}

Com o objetivo de estudar o desempenho das cultivares sob estresse de nitrogênio, essas foram representadas em um plano cartesiano por meio da relação entre a produção de matéria seca de raízes nas doses contrastantes de nitrogênio de acordo 
com Chun et al. (2005). A maioria das cultivares apresentou comportamento distinto quanto ao uso do $\mathrm{N}$, representados no gráfico pelas posições mais distantes da linha divisória (Figura 1). Assim, as cultivares que tiveram maiores pesos MSR em baixo nitrogênio foram DKB789 e o DKB 747, sendo esses, os mais promissores para solos com baixa disponibilidade desse nutriente. Para solos com maior disponibilidade de nitrogênio destacaram-se os híbridos DKB566, DKB747, DKB789, P30F53 e P30S40.

Figura 1. Eficiência nutricional de cultivares de milho para o peso de matéria seca de raiz, em condições de alto e baixo nitrogênio, de acordo com a metodologia proposta por Chun et al. (2005).

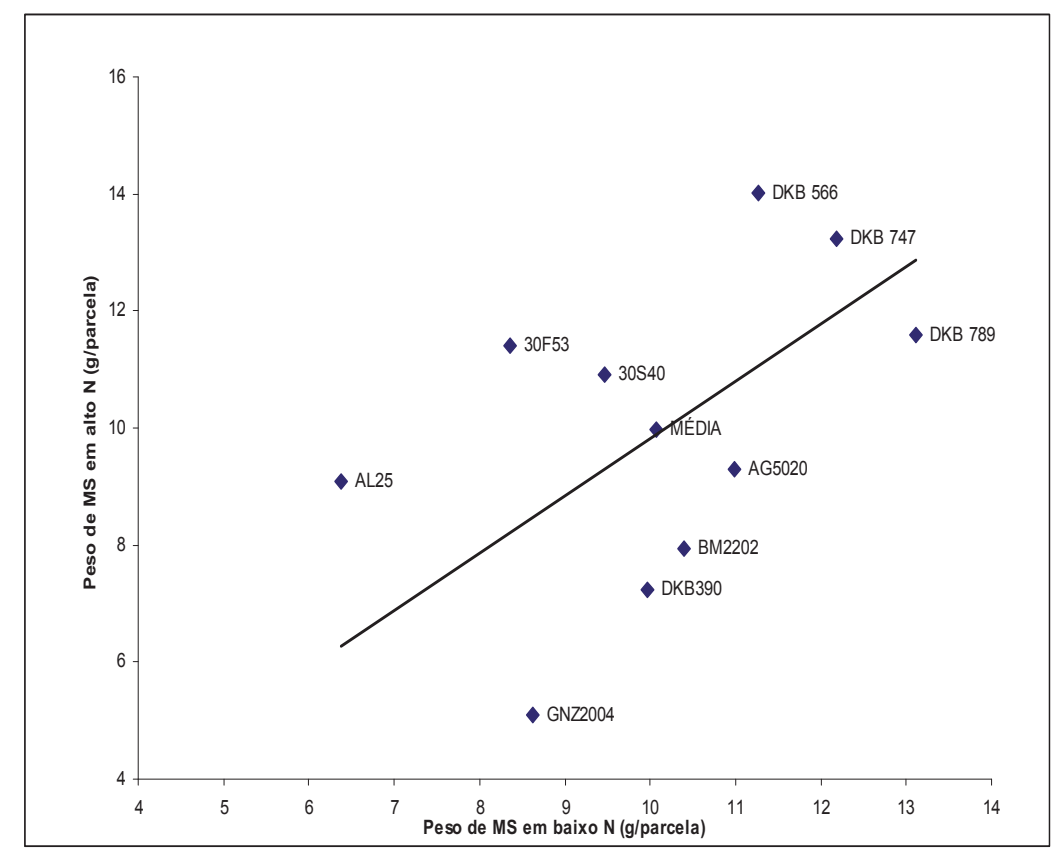

Fonte: Elaboração dos autores.

A metodologia proposta por Fageria e Kluthcouski(1980), permitiu identificar as cultivares com maiores pesos de MSR (DKB789, AG5020 e BM2202), portanto adaptadas exclusivamente às condições de estresses (Figura 2). As cultivares eficientes e responsivas, que apresentaram aumento no acúmulo de MSR em resposta ao aumento da disponibilidade de $\mathrm{N}$ e que possuem alta média de peso de MSR, superior à média do experimento em baixo N, foram a DKB747 e a DKB566. Esses cultivares estão adaptados ao estresse e respondem aos insumos aplicados.
As cultivares DKB566 e DKB747 foram as mais influenciadas pela disponibilidade de nitrogênio no solo, ou seja, obtiveram maiores pesos de MSR com alta adubação, sendo superado no ambiente com estresse de N pelo híbrido DKB 789.

As cultivares de milho eficientes no uso de nitrogênio foram: DKB566, DKB747, DKB789, AG5020 e BM2202; os responsivos ao fornecimento de N foram P30F53, DKB566, AL25, P30S40 e DKB747, evidenciando a existência de variabilidade entre cultivares para eficiência do uso e resposta à aplicação de N. 
Figura 2. Eficiência no uso e resposta à aplicação de nitrogênio em cultivares de milho, pela metodologia de Fageria e Kluthcouski (1980).

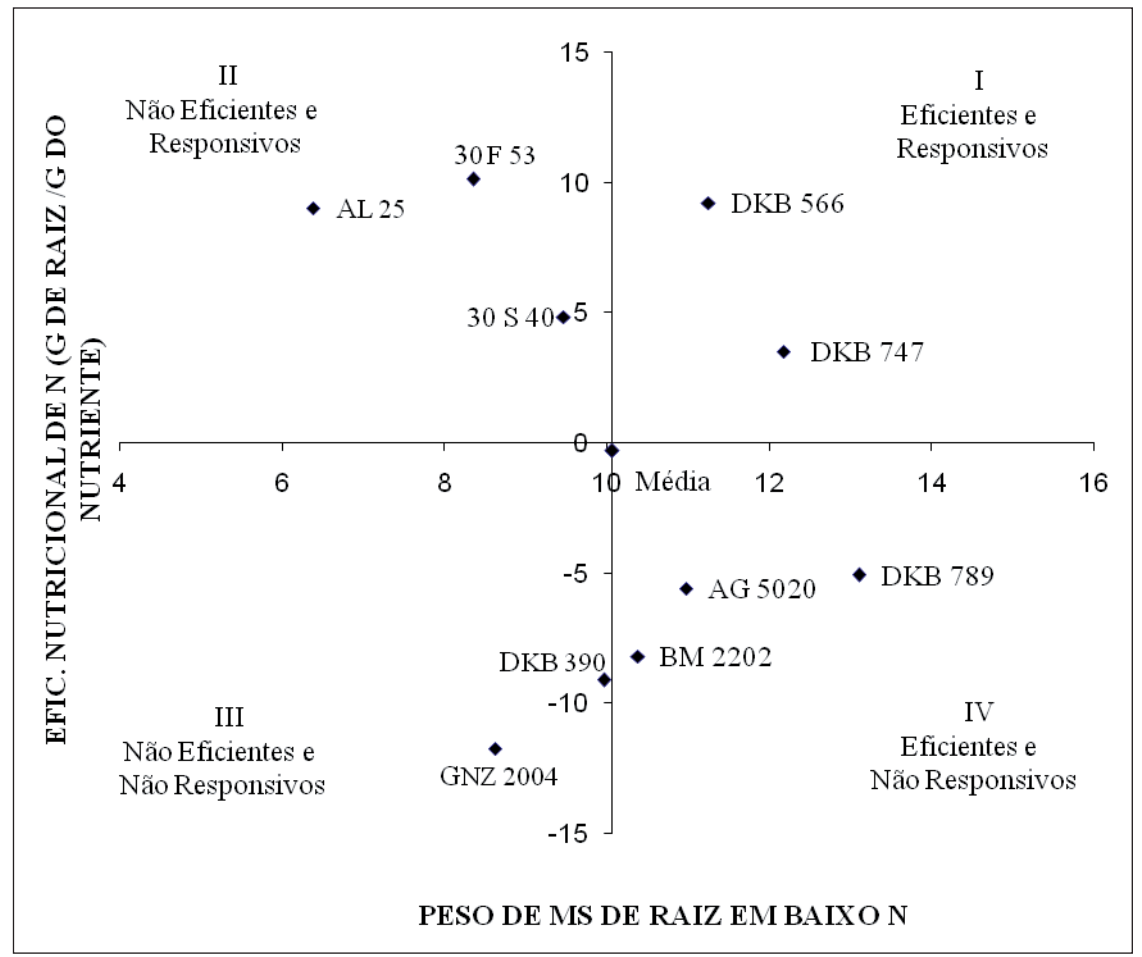

Fonte: Elaboração dos autores.

\section{Conclusões}

As cultivares de milho apresentaram características distintas quanto à eficiência no uso e absorção de nitrogênio nos dois ambientes estudados.

Genótipos eficientes para o uso do nitrogênio e responsivos a sua aplicação podem ser selecionados pela metodologia de Fageria e Kluthcouski (1980) em casa de vegetação.

Os níveis contrastantes de nitrogênio utilizados permitiram que ocorressem as diferenças necessárias, entre as características estudadas, para a seleção das cultivares mais eficientes.

\section{Agradecimentos}

Os autores agradecem ao $\mathrm{CNPq}$ (Conselho Nacional de Desenvolvimento Científico e Tecnológico) pelo suporte financeiro.

\section{Referências}

CHUN, L.; MI, G. H.; LI, J.; CHEN, F.; ZHANG, F. Genetic analysis of maize root characteristics in response to low nitrogen stress. Plant and Soil, The Hague, v. 276, n. 1-2, p. 369-382, 2005.

CLARK, R. B. Plant response to mineral element toxicity and deficiency. In: CHRISTIANSEN, M. N.; LEWIS, C. F. (Ed.). Breeding plants for less favorable environment. New York: J. Wiley, 1982. p. 71-142.

COOKE, G. W. Maximizing fertilizer efficiency by overcoming constraints to crop growth. Journal of Plant Nutrition, New York, v. 10, n. 9-16, n. 1, p. 1357-1369, 1987.

EMPRESA BRASILEIRA DE PESQUISA AGROPECUÁRIA - EMBRAPA. Centro Nacional de Pesquisa de Solos (Rio de Janeiro, RJ). Sistema brasileiro de classificação de solos. Brasília: EmbrapaSPI, Embrapa-CNPS, 1999. 412 p.

FAGERIA, N. K. Otimização da eficiência nutricional na produção das culturas. Revista Brasileira de Engenharia Agrícola e Ambiental, Campina Grande, v. 2, n. 1, p. 6-16, 1998. 
FAGERIA, N. D.; KLUTHCOUSKI, J. Metodologia para avaliação de cultivares de arroz e feijão para condições adversas de solo. Brasília: EMBRAPA/CNPAF, 1980. (Documentos, 8).

FERNANDES, F. C. S.; BUZZETI, S.; ARF, O.; ANDRADE, J.A. C. Doses, eficiência e uso do nitrogênio por seis cultivares de milho. Revista Brasileira de Milho e Sorgo, Sete Lagoas, v. 4, n. 2, p. 195-204, 2005.

FERREIRA, D. F. Programa estatístico SISVAR (Software). Lavras: UFLA, 1999.

FISHER, N. M.; DUNHAN, R. J. Root morphology and nutrient uptake. In: GOLDSWORTHY, P. R.; FISHER, N. M. (Ed.). The physiolgy of tropical fields crops. New York: J. Wiley, 1984. p. 25-117.

FÖHSE, D.; CLAASSEN, N.; JUNGK, A. Phosphorus efficiency of plants. I. External and internal P requeriment and $\mathrm{P}$ uptake efficiency of different plant species. Plant Soil, The Hague, v. 110, n. 1, p. 101-109, 1988.

GERLOFF, C. G. Plants efficiencies in the use of nitrogen, phosphorus and potassium. In: WRIGHT, M. J. (Ed.). Plant adaptation to mineral stress in problem soils. Ithaca: Cornell University, 1977. p. 161-173.

GODDARD, R. E.; HOLLIS, C. A. The genetic basics of forest tree nutrition. In: BOWEN, G. D.; NAMBIER, E. K. S. (Ed.). Nutrition of plantation forest. London: Academic, 1984. p. 237-258.

GOURLEY, C. J. P.; ALLAN, D. L.; RUSSELE, M. P. Plant nutrient efficiency: a comparison of definitions and suggested improvement. Plant and Soil, The Hague, v. 158, n. 1, p. 29-37, jan. 1994.

GRAHAM, R. D. Breeding for nutritional characteristics in cereals. In: TINKER, P. B.; LAUCHLI, A. (Ed.). Advances in plant nutrition. New York: Praeger, 1984. p. 57-102.

GUIMARÃES, L. J. M.; MARRIEL, I. E.; PARENTONI, S. N.; GUIMARÃES, C. T.; MIRANDA, G. V.; VASCONCELOS, M. J. V.; GAMA, E. E. G. Comportamento de linhagens de milho contrastantes no uso de nitrogênio cultivadas em dois níveis de $\mathrm{n}$ em substrato hidropônico. In: CONGRESSO NACIONAL DE MILHO E SORGO, 26., 2006, Belo Horizonte. Resumo expandido... Sete Lagoas: Associação Brasileira de Milho e Sorgo, 2006. p. 638.

ISRAEL, D. W.; RUFTY JUNIOR, T. W. Influence of phosphorus nutrition on phosphorus and nitrogen utilization efficiencies and associated physiological response in soybean. Crop Science, Madison, v. 28, n. 6, p. 954-960, nov./dec. 1988.

MALAVOLTA, E. Elementos de nutrição mineral de plantas. São Paulo: Ceres, 1980. 251 p.

MARANVILLE, J. W.; CLARK, R. B.; ROSS, W. M. Nitrogen efficiency in grain sorghum. Journal of Plant Nutrition, New York, v. 2, n. 5, p. 577-589, 1980.

MARRIEL, I. E.; FRANÇA, G. E.; VASCONCELLOS, C. A.; GAMA, E. E. G.; SANTOS, M. X.; OLIVEIRA, A. C. Eficiência de Absorção de Nitrogênio e Produtividade de Grãos em Populações de Milho Cultivadas Sob Estresse In: CONGRESSO NACIONAL DE MILHO E SORGO, 23., 2000, Uberlândia. Resumo expandido... Sete Lagoas: Associação Brasileira de Milho e Sorgo, 2000. 392 p.

MARTINEZ, H. E. P.; NOVAIS, R. F.; SACRAMENTO, L. V. S. do; RODRIGUES, L. A. Comportamento de variedades de soja cultivadas sob diferente níveis de fósforo: II. Translocação do fósforo absorvido e eficiência nutricional. Revista Brasileira de Ciência do Solo, Campinas, v. 17, n. 2, p. 239-244, maio/ago. 1993.

MELO, W. J. Dinâmica das formas de carbono e de nitrogênio em um Latossolo Roxo cultivado com Sorghum bicolor (L) Moench e com Dolichos lablab L, isoladamente, ou em cultura intercalada. 1977. Tese (Livre Docência) - Universidade Estadual Paulista, Jaboticabal.

MOLL, R. H.; KAMPRATH, E. J.; JACKSON, W. A. Analysis and interpretation of factors which contribute to efficiency of nitrogen utilization. Agronomy Journal, Madison, v. 74, n. 3, p. 562-564, may/jun. 1982.

PRESTERL, T.; SEITZ, G.; LANDBECK, M.; THIEMT, E. M.; SCHMIDT, W.; GEIGER, H. H. Improving nitrogen-use efficiency in European maize: estimation of quantitative genetic parameters. Crop Science, Madison, v. 43, n. 4, p. 1259-1265, 2003.

SCOTT, A. J.; KNOTT, M. A cluster analysis method for grouping means in the analysis of variance. Biometrics, Washington, v. 30, n. 3, p. 507-512, 1974.

YOUNGQUIST, J. B.; COX, P. B.; MARANVILLE, J. W. Evaluation of alternative screening criteria for selecting nitrogen-use efficient genotypes in sorghum. Crop Science, Madison, v. 32, n. 6, p. 1310-1313, nov./ dec. 1992. 
